\title{
Synthesis, Isolation and Crystal Structure Characterization of (Z)-4'-(1-Phenyl-2-p-tolylvinyl)biphenyl-4-carbaldehyde
}

\author{
Chul-Bae Kim, Hyunjong Jo, Sung-Kyung Lee, and Kwangyong Park* \\ School of Chemical Engineering and Materials Science. Chung-ing Lmiversitw, Seoul 156-756. Korea \\ ${ }^{\star E-m a i l: ~ K y p a r k a c a t . a c . k r}$ \\ Received June 23. 2009. Accepted September 3. 2009
}

Key Words: $(Z)-f^{\prime}-(1-P h e n y l-2-p$-tolỵlyinỵl)biphenyl-4-carbaldehỵdde. X-ray structure. Single crystal. NOESY spectra, Horner-Wadsworth-Emmons reaction

Fluorescent organic materials are used in a range of applications including bio-sensors. ${ }^{3}$ optical storage devices. ${ }^{-}$and organic solar cells. ${ }^{3}$ They have recently gained particular interest as efficient light emitters and charge transporters in organic light-emitting diodes (OLEDs) due to their significant advantages in energy consumption and flexible applications. ${ }^{4}$ The improved efficiency and stability of blue enitting materials is especially important in the area of OLEDs, because efficient blue emitters are rare due to the intrinsic wide band-gap. Blue fluorescent materials include oligopheny lenes, ${ }^{6}$ diarylanthracenes ' and poly'(pheny lene vinylene)s. Recently organometallic phosphorescent materials including iridium (III) complexes have been studied due to their high efficiency.

Since Hosokawa's pioneering work with 4.4 -bis(2,2-diphenylvinyl)-1. 1'-biphenyl (DPVBi) in the 1990s, "various distyrylarylenes (DSAs) have been investigated as representative blue fluorescent materials in OLEDs. " However. their low quantum efficiency. low glass transition temperature and crystallizing tendency have limited their applications in OLEDs. ${ }^{1 \geq}$ The tendency for cry stallization eventually degrades the lifetime of the corresponding devices by destroying the film homogeneity and crystal boundary. ${ }^{13}$ Structural modifications have been investigated to decrease the crystallizing tendency of DSAs, typically by constructing spiro-DSAs ${ }^{14}$ and tetrahedral DSAs.

The Horner-Wadsworth-Emmons reaction ${ }^{16}$ has been frequently used to prepare $\pi$-conjugated hydrocarbons for OLEDs and organic field-effect transistors. While $(E)$-isomers are predominantly produced by reactions of aldehydes with benzyl phosphonates. reactions of ketones generate mixtures of $(E)$ and $(Z)$-geometric isomers that are difficult to be resolved. ${ }^{\text {llbi, } 18}$ Significant problems in understanding the relationship between chemical stnictures and physical properties of emitting materials have been encountered because each isomer show's different optical properties. ${ }^{19}$ Therefore. an efficient separating process for two geometric isomers has been explored for the systematic development of functional $\pi$-conjugated hydrocarbons.

In a program for the development of blue fluorescent materials in our laboratory: 1-(4-bromophenyl)-2-(4-metlylphenyl)I-phenylethylene (3). an important intermediate for unsymmetrical DSAs. was prepared by the reaction of 4-bromobenzophenone (1) with diethỵl (4-methylbenzyl)phosphonate (2) (Scheme 1). However the crude product 3 was obtained as a 60 : 40 mixture of geometric isomers that were not separable by common chromatographic methods. Fortunately: we observed that a careful recry stallizing process in 2-propanol facilitated the selective isolation of both isomers. Nuclear nagnetic resonance (NMR) study showed that the initially recrystallized major product was (Z)-isomer, 3a. (Z)--'-(1- Phenyl-2-p-tolylvinyl)biphenyl-4-carbaldehydde (5) was prepared by SuzukiMiy aura reaction of 3 a with 4 -formy lphenylboronic acid (4), and recrystallized in $n$-hexane : $\mathrm{Et}_{2} \mathrm{O}(8: 1)$ to give a single crystal that was characterized by $\mathrm{X}$-ray structure analy sis. The results of this study are presented and discussed below.

The reaction of 1 with 2 in the presence of potassium tertbutoxide produced a $60: 40$ mixture of geometric isomers of bromotriphenylethỵlene 3 at the refluxing temperature of THF. While conventional cluromatographic methods were not able to resolve those isomers. the recrystallization process in 2-propanol facilitated the selective isolation of each isomer in a crystalline form. The major product 3 a was initially obtained as a clear crystalline solid. which was purified by a double recrystallizing process, in $31 \%$ isolated yield with $98 \%$ purity. The other isomer $3 \mathbf{b}$ was obtained by the consecutive recrystallization process of the remaining mother liquor.

The crystalline solids of both isomers were not uniform enough for single-crystal X-ray crystallography. Although various spectroscopic analyses were performed to distinguish the configuration of both isomers. their configuration could not be easily identified. Among ${ }^{1} \mathrm{H}-\mathrm{NMR},{ }^{13} \mathrm{C}-\mathrm{NMR}$, correla-
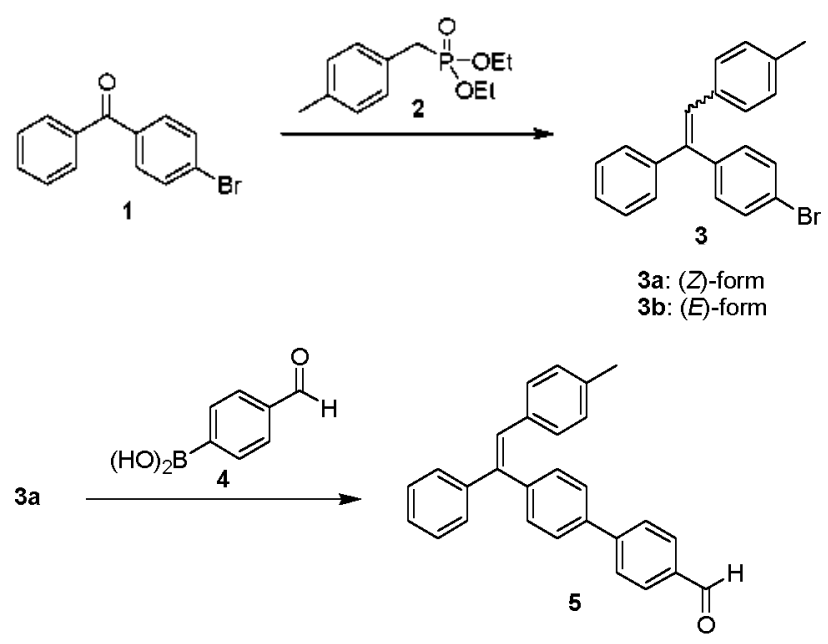

Scheme 1 

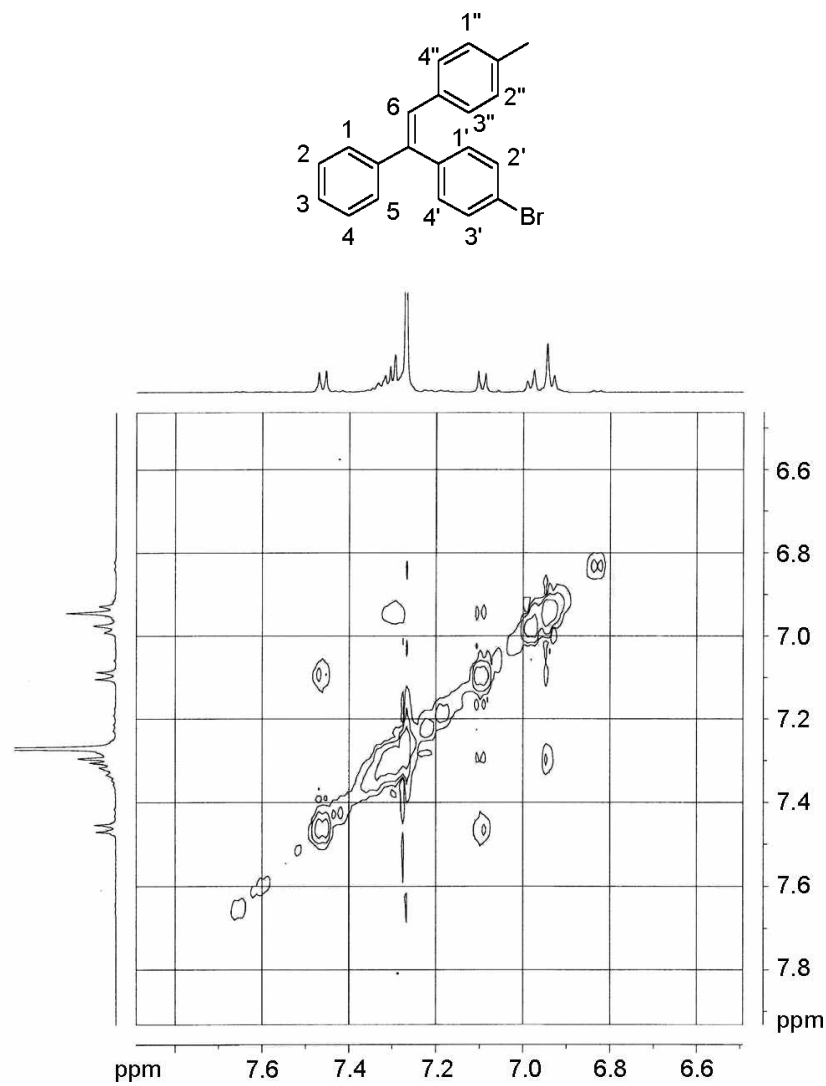

Figure 1. NOESY spectra of $\mathbf{3 a}$

tion spectroscopy (COSY), muclear Overhauser effect spectroscopy (NOESY) and heteronuclear single quantum coherence (HSQC) analyses analyses, the NOESY spectra of 3 a presented a vague clue (Figure 1). The correlations between $\mathrm{H}-6$ at $\tilde{\delta}$ 7.05 and $\mathrm{H}-1 \delta 7.30$ and between $\mathrm{H}-3^{\prime \prime}$ at $\delta 7.05$ and $\mathrm{H}-\mathrm{l}^{\prime}$ at $\delta$ 6.94 suggested that 3a may have the $Z$-configuration.

The configuration of 3 a could be clearly determined after the synthesis of $\mathbf{5}$. Stilbene $\mathbf{5}$ was prepared by the palladiumcatalyzed cross-coupling reaction of 3awith $\boldsymbol{4}$ in the presence of sodium carbonate. The crude product was purified by column chromatography to give 5 in $85 \%$ isolated yield. A single cry'stal of 5 was obtained by recry stallization of the purified product in $n$-hexane : $\mathrm{Et}_{-} \mathrm{O}(8: 1)$. The single-crystal X-ray cry stallography of 5 has not been disclosed before. The stnictural information of the crystalline 5 is presented in Table 1 .

The molecular stnicture of compound 5 illustrated in Figure 2 clearly shows the configuration and non-planar conformation of 5 . The torsion angles $\mathrm{C} 2-\mathrm{Cl}-\mathrm{Cl} 6-\mathrm{C} 18 . \mathrm{C} 2-\mathrm{Cl}-\mathrm{Cl} 3-\mathrm{C}$. and $\mathrm{C} 1-\mathrm{C} 2-\mathrm{C} 6-\mathrm{C} 10$ in 5 were $29.4(4) .57 .8(4)$. and $38.4(4)^{\circ}$, respectively. The bond length of $\mathrm{Cl}=\mathrm{C} 2$ was 1.353 (4) $\mathrm{A}$. which is longer than the reported $\mathrm{C}=\mathrm{C}$ bond lengths of trans $^{-1}{ }^{\prime \prime}$ and cis-stilbenes. ${ }^{\beth 1}$

The steric hindrance between rings $\mathbf{a}$ and $\mathbf{c}$ created a significant distortion around the ethylene group. and especially broadened the angle $\mathrm{C} 7-\mathrm{Cl} 14-\mathrm{Cl} 5$ to $127.9(2)^{\circ}$. The dihedral angles between rings $\mathbf{a}$ and $\mathbf{b}$. rings $\mathbf{a}$ and $\mathbf{c}$ and rings $\mathbf{a}$ and $\mathbf{d}$ were $36.6(2), 57.0(2)$, and $76.1(2)^{\circ}$, respectively (Figure 3 ). Among the six angles in phenyl rings. the angles involving C4. C6. C8. C13, and $\mathrm{Cl6}$. which are directly connected to
Table 1. Crystal data and structure refinement for 5

\begin{tabular}{|c|c|}
\hline Empirical formula & $\mathrm{C}_{28} \mathrm{H}_{22} \mathrm{O}$ \\
\hline Formula weight & 374.46 \\
\hline lemperature & $296(2) \mathrm{K}$ \\
\hline Crystal system, space group & Monoclinic, $P 2(1) / c$ \\
\hline Unit cell dimension & $\begin{array}{l}a=11.684(2) \AA \\
b=15.626(2) \AA \\
c=11.325(2) \AA \\
\beta=92.66(1)^{0}\end{array}$ \\
\hline Volume & $2065.4(5) \AA^{3}$ \\
\hline$Z$ & 4 \\
\hline Absorption coefficient & $0.07 \mathrm{~mm}^{-1}$ \\
\hline$F(000)$ & 792 \\
\hline 'l heta range for data collection & 2.18 to $28.61 \mathrm{deg}$ \\
\hline Index range & $\begin{array}{l}-15 \leq h \leq 15 \\
-21 \leq k \leq 20 \\
-13 \leq l \leq 15\end{array}$ \\
\hline Independent reflections & $5192[\mathrm{R}$ (int) $=0.069]$ \\
\hline Goodness-of-tit on $\mathrm{F}^{-}$ & 1.051 \\
\hline Final $R$ indices $[\mathrm{I}>2 \sigma(\mathrm{I})]$ & $\begin{array}{l}R=0.1303 \\
R_{w}=0.3223\end{array}$ \\
\hline Largest diff. peak and hole & 0.33 and $-0.42 \mathrm{e} / \AA^{3}$ \\
\hline
\end{tabular}

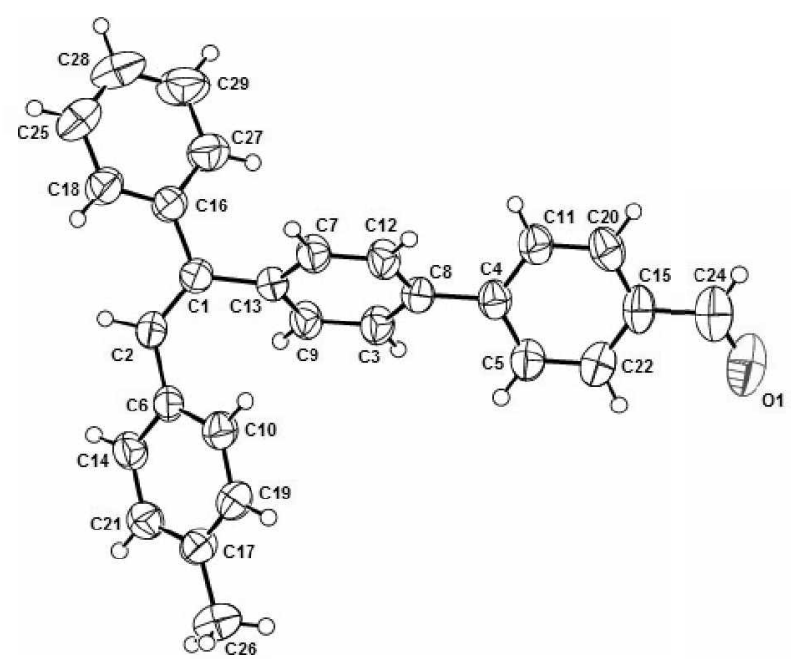

Figure 2. Molecular structure of $\mathbf{5}$, showing $50 \%$ probability displacement ellipsoids and the atom-numbering scheme.

outside the $s p^{\prime}$ carbon of the ring. were generally smaller than the others.

In summary. bromotriphenylethylene 3 was prepared as a $60: 40$ mixture of geometric isomers and efficiently separated to give both pure $(Z)$ - and $(E)$-isomers by a careful recrystallizing process in 2-propanol. NMR study revealed the initially recrystallized major product to be the (Z)-isomer, $3 \mathrm{a}$ The configuration of $3 a$ was confirmed by the first single crystal $X$-ray structure analysis of (Z)-4'-( l-phenyl-2-p-tolylvinyl)biphenyl4-carbaldehyde (5) that was prepared by Suzuki-Miyaura reaction of 3a with 4 and recry stallized in $n$-hexane : $\mathrm{Et}_{2} \mathrm{O}(8: 1)$. The procedure described in this paper is a promising synthetic route for preparing important intennediates of unsymmetrical DSAs. It is also expected to facilitate the systematic develop- 


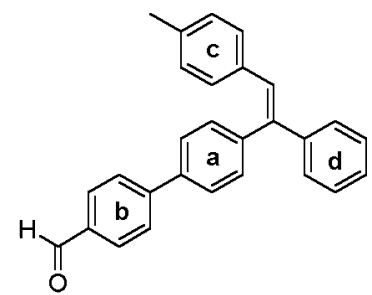

Figure 3. Molecular structure of 5 .

ment of finctional $\pi$-conjugated hydrocarbons. such as organic fluorescent materials.

\section{Experimental Section}

Preparation of 1-(4-bromophenyl)-2-(4-methylphenyl)-1phenylethylene (3). To the mixture of $2(35.1 \mathrm{mmol} .8 .51 \mathrm{~g})$ and potassium tert-butoxide $(54.0 \mathrm{mmol}, 6.06 \mathrm{~g})$ in THF (54 $\mathrm{mL})$ was added a solution of $1(27.0 \mathrm{mmol} .5 .00 \mathrm{~g})$ in THF $(200 \mathrm{~mL})$ at room temperature under an Ar atmosphere. The mixture was stirred at refluxing temperature for $3 \mathrm{~h}$. The reaction mixture was cooled to room temperature; diluted with ethyl acetate $(300 \mathrm{~mL})$ : washed with $1 \%$ aqueous $\mathrm{HCl}$ $(200 \mathrm{~mL})$, water $(300 \mathrm{~mL})$ and brine: dried over $\mathrm{MgSO}_{4}$ : and concentrated in vacuo. When the crude product was purified by column chromatography 3 was obtained as a white powder $(8.83 \mathrm{~g}, 94 \%$ ) that was a $60: 40$ mixture of $3 \mathrm{a}$ and $3 \mathrm{~b}$ by GC analysis. When the mixture was recrystallized from 2-propanol. a crystalline product was generated as a $91: 9$ mixture of $\mathbf{3} \mathbf{a}$ and $\mathbf{3 b}$. This crystalline mixture was purified further by the second recrystallization from 2-propanol to give $\mathbf{3 a}$ as a needle crystal with $98 \%$ purity $(2.91 \mathrm{~g} .31 \%)$ : TLC $R_{\mathrm{f}} 0.73$ (n-hexane : $\mathrm{Et}_{2} \mathrm{O}=1: 1$ ), mp $119-121^{\circ} \mathrm{C}$. ${ }^{1} \mathrm{H}$ NMR spectnum $\left(500 \mathrm{MHz}_{2} \mathrm{CDCl}_{3}\right)$ ò $2.28(\mathrm{~s}, 3 \mathrm{H}) .6 .93(\mathrm{~d}, J=8.0 \mathrm{~Hz} .2 \mathrm{H})$. $6.93(\mathrm{~s} .1 \mathrm{H}) .6 .97(\mathrm{~d} . J=8.0 \mathrm{~Hz}, 2 \mathrm{H}) .7 .09$ (d. $J=8.3 \mathrm{~Hz} .2 \mathrm{H})$. 7.27-7.31 (m. 5H). 7.45 (d. $J=8.3 \mathrm{~Hz} .2 \mathrm{H}):{ }^{13} \mathrm{C} \mathrm{NMR}$ spectrum (125 MHz. $\left.\mathrm{CDCl}_{3}\right)$ ò 21.4, 121.6, 127.8(×3). 128.5(×2). 128.9, $129.1(\times 2), 129.7(\times 2), 132.1(\times 2), 132.5(\times 2), 134.4,137.1$. 139.8, 140.7, 143.3: HRMS (EI. 70 eV) calcd for $\mathrm{C}_{21} \mathrm{H}_{1}: \mathrm{Br}$ (M) 348.0514 . found 348.0495

The $(E)$-isomer $3 \mathrm{~b}$ was obtained by the consecutive recrystallizing process of the remaining mother liquor. Recrystallization of the white solid that was obtained by concentrating the remaining solution produced a pure crystalline $3 \mathrm{~b}(0.281 \mathrm{~g}$. $3 \%$ ): TLC $R_{\mathrm{f}} 0.73$ (n-hexane : $\left.\mathrm{Et}_{2} \mathrm{O}=1: 1\right), \mathrm{mp} 69-70^{\circ} \mathrm{C}$. ${ }^{1} \mathrm{H}$ NMR spectrum $\left(500 \mathrm{MHz}, \mathrm{CDCl}_{3}\right)$ ò $2.26(5,3 \mathrm{H}) .6 .89-6.91$ (m. $3 \mathrm{H}) .6 .94(\mathrm{~d}, J=8.1 \mathrm{~Hz} .2 \mathrm{H}) .7 .16-7.19(\mathrm{~m} . \mathrm{H}) .7 .33-7.34$ (m. 3H) $.7 .4 \mathrm{l}(\mathrm{d} . J=8.5 \mathrm{~Hz}, 2 \mathrm{H}),{ }^{13} \mathrm{C}$ NMR spectnum $(75$ $\left.\mathrm{MHz} \mathrm{CDCl}_{3}\right)$ ò 21.3, 121.6. 127.8. 128.8. 129.0(×4), 129.4 $(\times 2), 129.7(\times 2), 130.6(\times 2), 131.5(\times 2), 134.5,137.2,140.3$. 140.8, 142.8: HRMS (EI, 70 eV) calcd for $\mathrm{C}_{21} \mathrm{H}_{1}: \mathrm{Br}\left(\mathrm{M}^{+}\right)$. 348.0514 . found $348.05+0$.

Preparation of +'-(1-phenyl-2-p-tolyl-vinyl)-biphenyl-tcabaldehyde (5). To a solution of $3 \mathrm{a}(1.4 \mathrm{mmol}, 0.50 \mathrm{~g})$ and $\mathrm{Pd}\left(\mathrm{PPh}_{3}\right)_{4}(0.072 \mathrm{mmol}, 0.083 \mathrm{~g})$ in DMF $(60 \mathrm{~mL})$ was added $2.0 \mathrm{M}$ aqueous $\mathrm{Na}_{2} \mathrm{CO}_{3}(1.5 \mathrm{~mL})$. To the resulting mixture was added 4 ( $1.7 \mathrm{mmol}, 0.25 \mathrm{~g}$ ). which was dissolved in $\mathrm{MeOH}$ ( $1 \mathrm{~mL}$ ). The mixture was stirred at refluxing temperature for $7 \mathrm{~h}$.
The reaction nixture was cooled to room temperature; diluted with ethyl acetate $(150 \mathrm{~mL})$ : washed with $1 \%$ aqueous $\mathrm{HCl}$ $(100 \mathrm{~mL})$ : water $(300 \mathrm{~mL})$ and brine: dried over $\mathrm{MgSO}_{4}:$ and concentrated in vacuo. The crude compound was purified by column chromatography ( $n$-hexane : $\mathrm{Et}_{2} \mathrm{O}=4: 1$ ) to generate $5(0.445 \mathrm{~g} .85 \%)$ as a white solid: TLC $R_{0} 0.63$ (n-hexane : $\mathrm{Et}_{2} \mathrm{O}=$ $1: 1)$, mp $207-208^{\circ} \mathrm{C}$. ${ }^{1} \mathrm{H}$ NMR spectrum (500 MHz, $\mathrm{CDCl}_{3}$ ) $\delta 2.28$ (s. 3H), 6.96-6.99 (m. $5 \mathrm{H}) .7 .32-7.35(\mathrm{ml}, 7 \mathrm{H}), 7.63(\mathrm{~d}$, $J=8.2 \mathrm{~Hz}, 2 \mathrm{H}) .7 .82(\mathrm{~d}, J=8.2 \mathrm{~Hz}, 2 \mathrm{H}) .7 .97(\mathrm{~d}, J=8.2 \mathrm{~Hz}$, 2H). 10.07 (s. IH): ${ }^{13} \mathrm{C}$ NMR spectrum $\left(75 \mathrm{MHz} \mathrm{CDCl}_{3}\right) \delta$ $21.2,127.7(\times 4), 127.9(\times 2), 128.5(\times 2), 129.1,129.1(\times 2)$, $129.7(\times 2) .130 .6(\times 3), 131.5(\times 2) .134 .6,135.5,137.1 .138 .6$. 141.3, 141.3. 143.6, 146.9. 192.3: HRMS (EI, 70 eV) calcd for $\mathrm{C}_{28} \mathrm{H}_{2} \mathrm{O}\left(\mathrm{M}^{+}\right), 374.1671$, found 374.1670 . The product was recrystallized from $n$-hexane : $\mathrm{Et}_{-} \mathrm{O}(8: 1)$ to give a single crystal of 5 that was analyzed by X-ray crystallography.

$X$-ray crystallographic analysis for 5 . A single crystal of 5 was mounted on a glass fiber. The diffraction data for $\mathbf{5}$ were collected on a Bnuker IK SMART CCD-based diffractometer with graphite-monocluromated Mo-K $\alpha$ radiation $(\lambda=0.7107$ $\AA)$ at $296 \mathrm{~K}$. The reflection data were collected as $\varphi$ and $\omega$ scans. Cell parameters were determined and refined by the SMLART progran.." Data reduction and empirical absorption correction were performed using $S H N T$ software ${ }^{23}$ and the $S+1 D A B S$ program, ${ }^{24}$ repectively

Supplementary Data. Christallographic data for the structure 5 reported here have been deposited with the Cambridge Crystallographic Data Centre (Deporsition No. CCDC-734302). That data can be obtained free of charge via htp w'w w ccdc. cam ac.ukdata request cif (or from The CCDC, 12 Union Road. Cambridge CB2 lEZ. UK: fax: +4+ 1223336033 e-mail: depositáaccdc.cam.ac.uk).

Aclonowledgments. This research was supported by the Chung-Ang University Research Grants in 2009.

\section{Refeiences}

1. For reviews, see: (a) Nolan, E. M.; Lippard, S. L. Acc. Chem. Res. 2009, 12, 193. (b) Gonçalves, M. S. T. Chent. Rev. 2009, 109,190 . (c) Jose, J.; Burgess, K. Tetrihedron 2006, 62, 11021 . (d) Wang, F.: Tan, W. B.; Zhang, Y.: Fan, X.: Wang, M Nonotechol. 2006, $17, \mathrm{Rl}$

2. For reviews, see: (a) Oliveira, O. N., J⿸厂 Ir; Dos Santos, D. S., Jr.; Balogh, D. T: Zucolotto, V; Mendonça, C. R. Ads. Colloid Interface Sci. 2005, 116, 179. (b) Cappitelli, F.; Sorlini, C. Crit. Rev. Microbiol $\mathbf{2 0 0 5}, 31,1$.

3. For reviews, see: (a) Li, G.; Shrotriya, V:; Yao, Y.: Huang, T.; Yang. Y.J. Mater. Chem. 2007, 17,3126. (b) Gunes, S.; Neugeballer, H.: Sariciftci, N. S. Chem. Rev. 2007, 107, 1324. (c) Benanti, T. L.; Venkataraman, D. Photosynth. Res. 2006, 87, 73. (d) Coakley, K. M.; McGehee, M. D. Chent. Hater. 2004, 16, 4533 .

4. For reviews, see: (a) Kalinowski, I. Opt. Mater. 2008, $30,792$. (b) Geffroy, B.: le Roy, P.; Prat, C. Polm Int 2006, 55, 572. (c) Veinot, J. G. C.; Marks, T. I. Acc. Chemt Res. 2005, 38, 632.

5. (a) Shil, P.-I; Shu, C.-F ; Tung, Y.-L.; Chi, Y. Appl. Phys. Lett. 2006, 88, 251110 . (b) Raghunath, P.; Reddy, M. A.; Gouri, C.; Bhanuparakash, K.: Rao, V. J. J. Phus Chem. A 2006, 110, 1152. (c) Wen, S.W.; Lee, M-T.; Chen, H. C. J. Displon Technol 2005, 1,90 . 
6. (a) Cocherel, N.: Poriel, C.: Rault-Berthelot, I.; Bartière, F.: Audebrand, N.; Slawin, A. M. Z.: Vignau, L. Chem. Enr. J. 2008, 14, 11328. (b) Spelur, T.: Siebert, A.: Fulnmanu-Lieker, T.: Salbeck, J.; Rabe, T.; Riedl, T.; Johannes, H. H.; Kowalsky, W.; Wang, J.; Weimann, T.; Hinze, P. Appl Phes. Lett. 2005, 87, 161103. (c) Schneider, D.: Rabe, T.: Riedl, T.; Dobbertin, T:: Kroger, M.; Becker, E.: Tohannes, H. H.; Kowalsky, W.: Weimann, T.; Wang, T.: Hinze, P. J. Appl. Phos. 2005, 98, 043104. (d) Johansson, N.; Salbeck, T.; Baller, T.; Weissörtel, F.; Bröms, P.: Andersson, A.: Salaneck, W. R. Adw . Mater. 1998, 10, 1136.

7. (a) Jarikov, V. V. Appl. Phys. Lett. 2008,92, 244103. (b) Zelum, D.: Fudikar, W.: Hans, M.; Schilde, U.; Kelling, A.; Linker, T, Chent. Euf, J. 2008, 1t, 11429. (c) Martin, R.: Tenuel, L.: Aprile, C.: Cabeza, I. F.: Alvaro, M.: García, H. Tetrahedron 2008, 64, 6270. (d) Martín, R: Benitez, M: Cabeza, J. F.: García, H.: Leyva, A. J. Phws. Chem. $\mathrm{C}$ 2007, 111,7532 .

8. For reviews, see: (a) Grimsdale, A. C.; Chan, K. L.; Martin, R. E.; Jokisz, P. G.; Holmes, A. Chen. Rev. 2009, 109. 897. (b) Praven, V. K.: Babu, S. S.: Vijayakumar, C.: Varghese, R.: Ajayaghosh, A. Bull. Chem. Soc. Jphi 2008, 81, 1196. (c) Babudri, F.; Farinola, G. M: Naso, F. J. Hater. Chem. 2004, I H, I1. (d) Yamamoto, T. Afacromol Rapid Comnnu. 2002, 23, 583.

9. (a) Rausch, A. F.; Thompson, M. E.: Yersin, H. lhorg. Chem. 2009, 48, 1928. (b) Su, S.-J.: Takahashi. Y.: Chiba, T.: Takeda. T.; Kido, T. Adv. Funct A fater 2009, 19, 1260. (c) Xu, Y : Yang, R.: Peng, T.: Mikhailovsky, A. A.: Cao, Y: Nguven, T.-Q.; Bazan, G. C. Adv. Afoter: 2009, 21, 584. (d) Chen, F.-C.: Chien, S.-C.: Chen, Y.-S. Appl. Phvs. Lett. 2009, 94, 043306.

10. (a) Hosokawa, C.: Higashi, H.; Nakamura, H: Kusunnoto, T. Appl. Phy's. Lett. 1995, 67, 3853. (b) Hosokawa, C.; Tokailin, H.; Higashi, H.; Kusumoto, T. J. Appl. Phy's. 1995, 78, 5831. (c) Hosokawa, C.; Higashi, H.; Kusumoto, T. Appl Phvs. Lett. $1993,62,3238$.

11. (a) Williams, R. T.: Hodge, P.; Yeates, S. G. Polmt .Adv. Techol. 2008, 19, 569. (b) Williams, R. T; Hodge, P.; Yeates, S. G. React. Fmct. Polmm. 2007, 67, 1061. (c) Su, H.-I.: Wu, F.-I.; Tseng, Y.-H.: Shu, C.-F. Adv. Funct. Mater. 2005, 15, 1209. (d) Chen, Y.: Lai, S.-P. J. Polm Sci. Part A: Polym. Chem. 2001, 39, 2571

12. (a) Fischer, A.; Chenais, S; Forget, S.; Castex, M.-C. ; Adès, D.: Siove, A.: Denis, C.: Maisse, P.: Geffroy, B. J. Phws. D. Appl. Phys 2005, 39. 917. (b) Wu. F.-I.: Shih. P. I.: Yuan. M.-C.: Dixit, A. K.: Shu, C.-F.: Chung, Z.-M.; Diau, E. W.-G. J. Hater: Chem. 2005, 15, 4753. (c) Liao, C.-H.; Lee, M.-T.; Tsai, C.-H.: Chen, C. H. Appl Phvs. Lett. 2005, 86, 203507.

13. Joswick, M. D.: Campbell, I. H.; Barashkov, N. N.: Ferraris, J. P. J. Appl. Phys. 1996, 80,2883
14. Lill, S.: He, F.: Wang, H.: Xu, H.: Wang, C.: Li, F.: Ma, Y. J. Nater Chent $2008,18,4802$.

15. Wang, S.; Oldham, W. J.; Hudack, R. A.; Bazan, G. C. J. Am. Chem. Soc. 2000, 122, 5695.

16. Wadsworth, W. W.; Emmons, W. D. J.Am. Chem Soc. 1961,83, 1733

17. (a) Rigamonti, L.; Babgi, B.; Cifuentes, M. P.; Roberts, R. L.; Petrie, S.: Stranger, R.: Righetto, S.: Teshome, A.: Asselberghs, I.: Clays, K.: Humphrey, M. G. Inorg. Chem 2009, 48, 3562 (b) Snyder, S. A.; Breazzano, S. P.; Ross, A. G.; Lin, Y.; Zografos, A. L. 2009, 131, 1753. (c) Babu, S. S.; Praven, V. K; Prasanthkumar, S.; Ajayaghosh. A. Chem. Em. J. 2008, 14, 9577 . (d) Kábir, M. S.; Engelbrecht, K.; Polanowski, R; Krneger, S. M.; Ignasiak, R: Rott, M; Schwan, W. R:- Stemper, M. E:- Reed, K. D.; Sheman, D. Cook, T. M. Bioorg. Hed. Chent Lett. 2008, 18, 5745. (e) Feng, X.; Feng, F.: Yu, M.: He, F.: Xu, Q.: Tang, H.: Wang, S.; Li, Y.: Zhu, D. Org. Lett. 2008, 10,5369. (f) McGrier, P. L.; Solntsev, K. M.; Miao, S.; Tolbert, L. M.; Miranda, O. R:; Rotello, V. M.; Bunz, U. H. F. Chem. Eur, J. 2008, 14,4503 . (g) Cho, C.-H.; Park, K. Bull. Korean Chem. Soc. 2007, 28, 1159.

18. (a) Kim, C.-B.; Cho, C.-H.: Chai, K. Y.: Park, K. Acta Cnst. E 2008, E64, 0457. (b) Büttner, M. W.; Burschla, C.; Daiss, J. O; Ivanova, D.; Rochel, N.; Kamunerer, S.; Peluso-Iltis, C.; Bindler, A.: Galldon, C.: Germain, P.: Moras, D.; Gronemeyer, H.; Tacke, R. ChemBioChem 2007, 8, 1688 . (c) Leonik, F. M.; Papke, R. L.; Horenstein, N. A. Bioorg. Med. Chem. Lett. 2007, 17, 1520 . (d) Pander, R. K.; Wabharkar, R. D.; Kumar, P. Symth. Commm 2005, 35, 2795. (e) Femandez-Gacio, A.; Vitale, C.; Mouriño, A. J. Org. Chem. 2000, 65, 6978.

19. Ginocchietti, G.; Galiazzo, G.; Pannacci, D.; Mazzucato, U.; Spalletti, A. Chem. Phws. 2006, 331, 164.

20. (a) Gao. L.: Peng. H.: He. H.W. Acta Cnust. E 2006. E62. 05032. (b) De Borger, R.; Vande Velde, C. M. L.; Blockhuys, F. Acta Chst. E 2005, E61, 0819. (c) Ogawa, K.; Sabo, T.; Yoshimura, S. Takeuchi, Y: Toriumi, K. J. Ant. Chent. Soc. 1992, 114, 1041 .

21. (a) Bames, J. C.: Chuldek. J. A. Acta Cinst. E 2002. E58, 0603. (b) SethuSankar, K.; Saravanan, S.; Velmurugan, D.; Parvez, M. Acta Chst. C 2003, C59, ol 56.

22. SMLART, Version 5.0, Datá Collection Software, Bruker AXS Inc. Madison. WI, 1998.

23. SAINT, Version 5.0, Data Integration Software, Bruker AXS Inc.. Madison, WI, 1998.

24. Sheldrick, G. M. S.AD.ABS, Program for Absorption Correction with Bruker SMART system, Universitat Göttingen, Germany, 1996. 